\title{
Malignant Phyllodes Tumor of the Breast: A Case Report
}

\author{
Mohammed Al-Zoubaidi ${ }^{1}$, Suimin Qiu ${ }^{2,5}$, Mark Bonnen ${ }^{6}$, Melissa Joyner ${ }^{3}$, Kendall Roehl ${ }^{4}$, \\ Colleen Silva ${ }^{1}$ and Celia Chao ${ }^{*}, 1,5$
}

\author{
Departments of ${ }^{1}$ Surgery, ${ }^{2}$ Surgical Pathology, ${ }^{3}$ Radiation Oncology, ${ }^{4}$ Plastic Surgery, ${ }^{5}$ Sealy Center for Cancer Cell \\ Biology, University of Texas Medical Branch, Galveston, Texas, USA; ${ }^{6}$ Department of Radiation Oncology, Brazosport \\ Regional Health System, Lake Jackson, Texas, USA
}

\begin{abstract}
Phyllodes tumors of the breast have biphasic histological features with both epithelial and stromal components. Careful characterization of the stromal compartment is critical since it is the pathologic features of the stromal cells that determine its malignant potential. We present a case report of a woman with an aggressive malignant phyllodes tumor with sarcomatous differentiation, who succumbed to metastatic disease within 8 months of initial presentation.
\end{abstract}

Keywords: Spindle cell tumors, high-grade sarcoma component, malignant phyllodes, malignant fibrous histiocytoma, osteosarcoma.

\section{INTRODUCTION}

Phyllodes tumors (PT), previously called cystosarcoma phyllodes, are fibroepithelial tumors of the breast, representing $2-3 \%$ of all fibroepithelial tumors, and less than $1 \%$ of all breast tumors [1]. PT are uniquely found in breast tissue and histologically have both an epithelial component as well as metaplastic mesenchymal (stromal) component. Clonal analyses have revealed that some PT develop from fibroadenomas [2]. At least $12.5 \%$ of patients with PT have a history of fibroadenomas and over $20 \%$ of patients have a concurrent diagnosis of a benign fibroadenoma [3]. The pathological appearance of stromal overgrowth, stromal cellularity, degree of nuclear atypia, number of mitotic cells, and the nature of the tumor margin (circumscribed, pushing, or infiltrative) determines whether a tumor is a benign fibroadenoma, a benign PT, a borderline PT, or a malignant PT. The stroma from malignant PT are distinguished by marked cellularity with nuclear atypia, nuclear pleomorphism, increased mitotic activity, and stromal cell overgrowth [4, 5]. Heterogeneous stromal components are seen in PT and is commonly associated with malignant PT $[4,6,7]$. Malignant PT has a 5- and 10 -year overall survival of $84 \%$ and $77 \%$ after surgical extirpation [8].

In the present report, we discuss a patient who presented with a malignant PT with sarcomatous elements. She developed multiple large pulmonary metastases and brain metastases and expired within 8 months of initial presentation and diagnosis.

\section{CASE REPORT}

A 59-year-old Hispanic female was referred to our breast clinic for evaluation of a large left breast mass, which had been palpable for the past three years. The breast mass began

*Address correspondence to this author at the Department of Surgery, University of Texas Medical Branch, 301 University Boulevard, Galveston, Texas 77555, USA; Tel: 409-772-0698; Fax: 409-772-0088;

E-mail: cechao@utmb.edu to rapidly increase in size and became painful in the past year, and within the past 3 months prior to consultation, the breast mass grew significantly larger and eroded through the overlying skin. A core needle biopsy from an outside hospital reported a "malignant phyllodes tumor". She was postmenopausal, Gravida 5 Para 5, with a family history of breast cancer in her two maternal aunts who both died of the disease at 40 and 50 years of age, respectively.

On physical examination, she had a left $20 \times 20 \mathrm{~cm}$ multilobulated breast mass visible on the left side with erosion through the skin medially (Fig. 1). The contralateral breast examination was unremarkable. There was no palpable cervical, supraclavicular, or axillary lymphadenopathy. There was no clinical suspicion for distant disease. She underwent a total mastectomy with en-bloc partial pectoralis major muscle resection to ensure an adequate resection margins; a fasciocutaneous advancement flap was performed to achieve wound closure. She had an uneventful recovery and she was discharged on postoperative day two.

Final pathology report confirmed a malignant phyllodes breast tumor measuring $24 \mathrm{~cm}$ in greatest dimension; on cut surface, there was white and tan tissue with areas of hemorrhage and necrosis (Fig. 2). Microscopically, a leaflike pattern, characteristic of phyllodes, was seen focally at the edge of the tumor (Fig. 3A). The tumor consisted of mainly high-grade sarcoma with hypercellularity and necrosis (Fig. 3B). The heterologous components were comprised mainly of high-grade sarcoma (malignant fibrous histiocytoma-like, Fig. 3C) with high mitotic index (16 mitoses/10 HPF, Fig. 3C), osteosarcoma (5\%, Fig. 3D) and chondrosarcoma $(<5 \%$, not shown). There was no evidence of lymphovascular invasion, and all resection margins were free of the tumor. Two enlarged lymph nodes, found intraoperatively, were removed with the specimen, and were negative for malignancy on histopathology. 


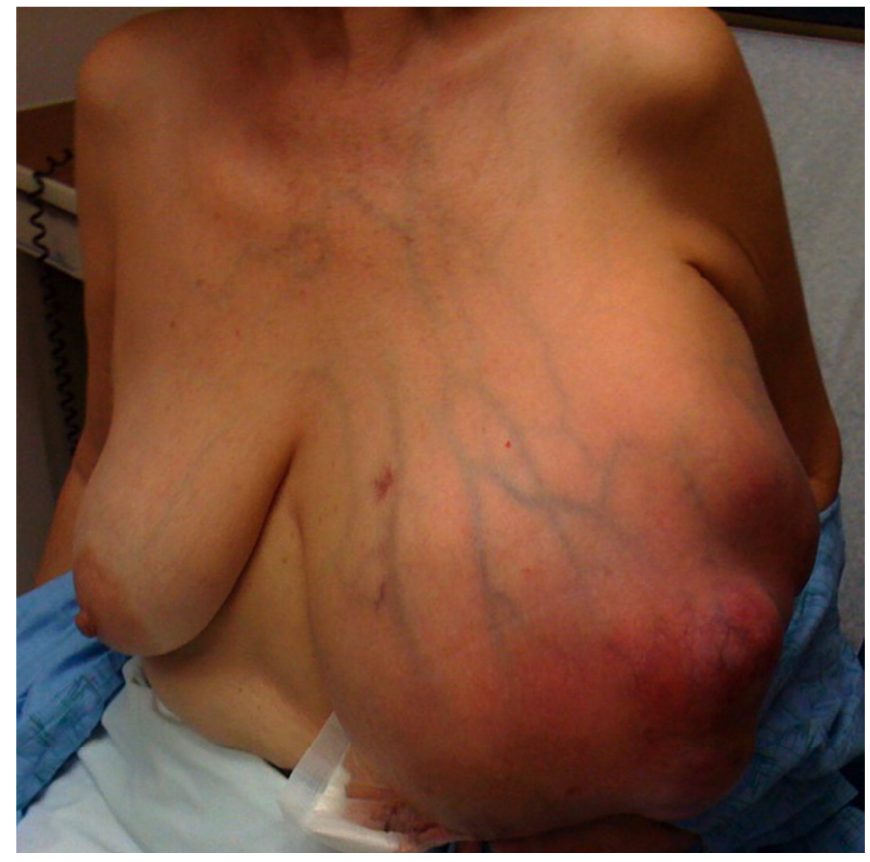

Fig. (1). The patient with a very large left breast mass with prominent, enlarged veins overlying the tumor, at initial clinical presentation.

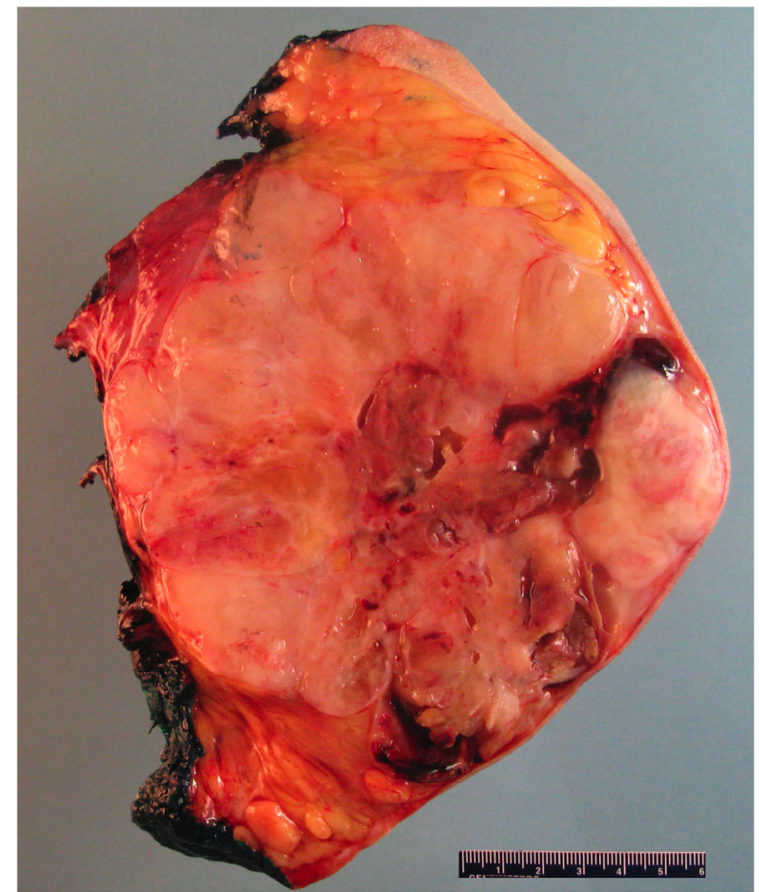

Fig. (2). Cross section of the tumor revealing a white-tan fleshy tumor with focal areas of necrosis and hemorrhage.
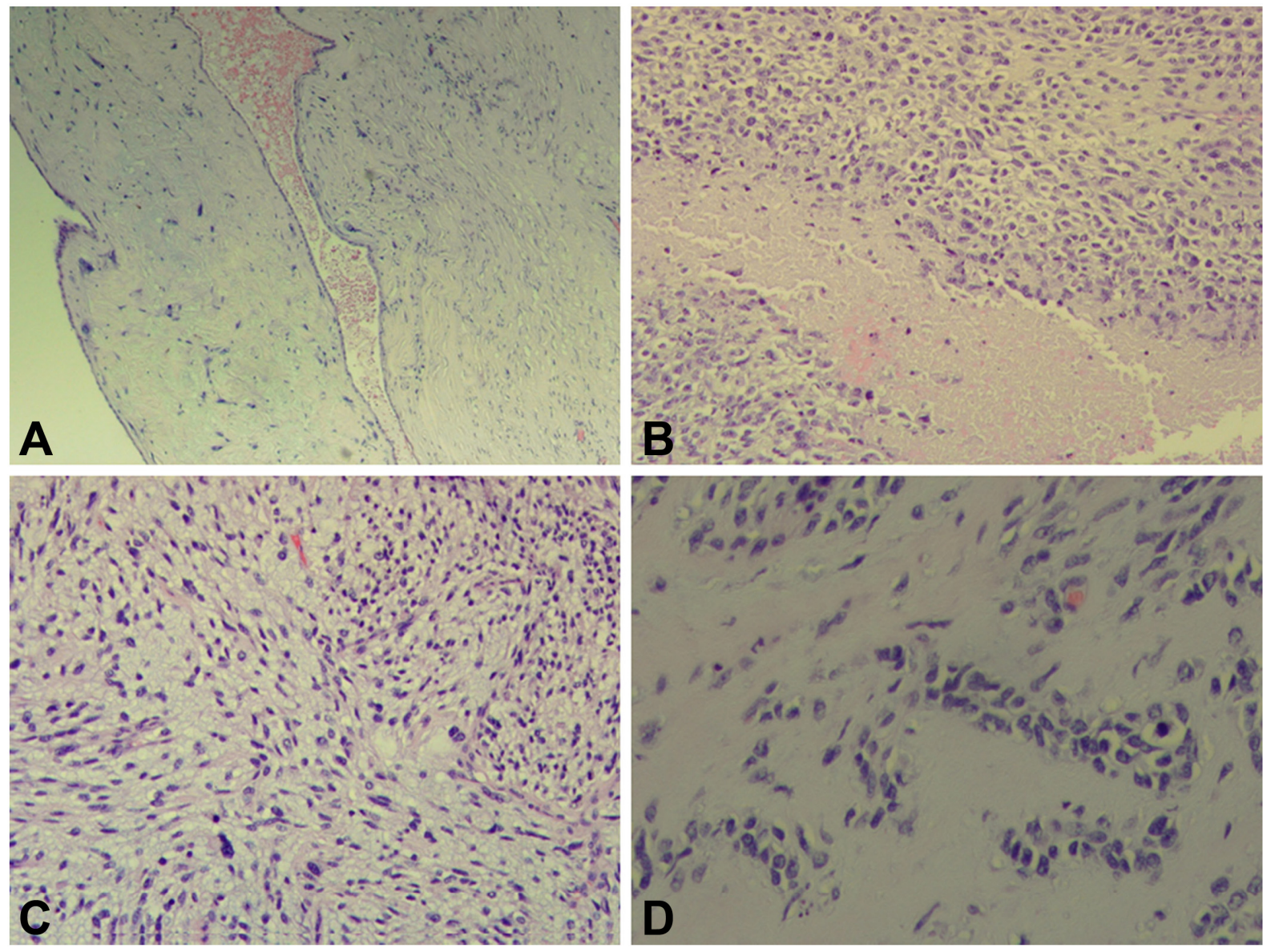

Fig. (3). Histologic features of the tumor. (A) Areas with phyllodes or leaf-like pattern with characteristic biphasic features: epithelial and stromal components. (B) The overgrowth of the high-grade sarcoma has extensive coagulative necrosis, and (C) hypercellular spindle cell proliferation with nuclear atypia and active mitotic figures. (D) Malignant sarcoma with osteoid production was in a portion of the tumor, indicating the presence of an osteosarcomatous component in this malignant PT. 

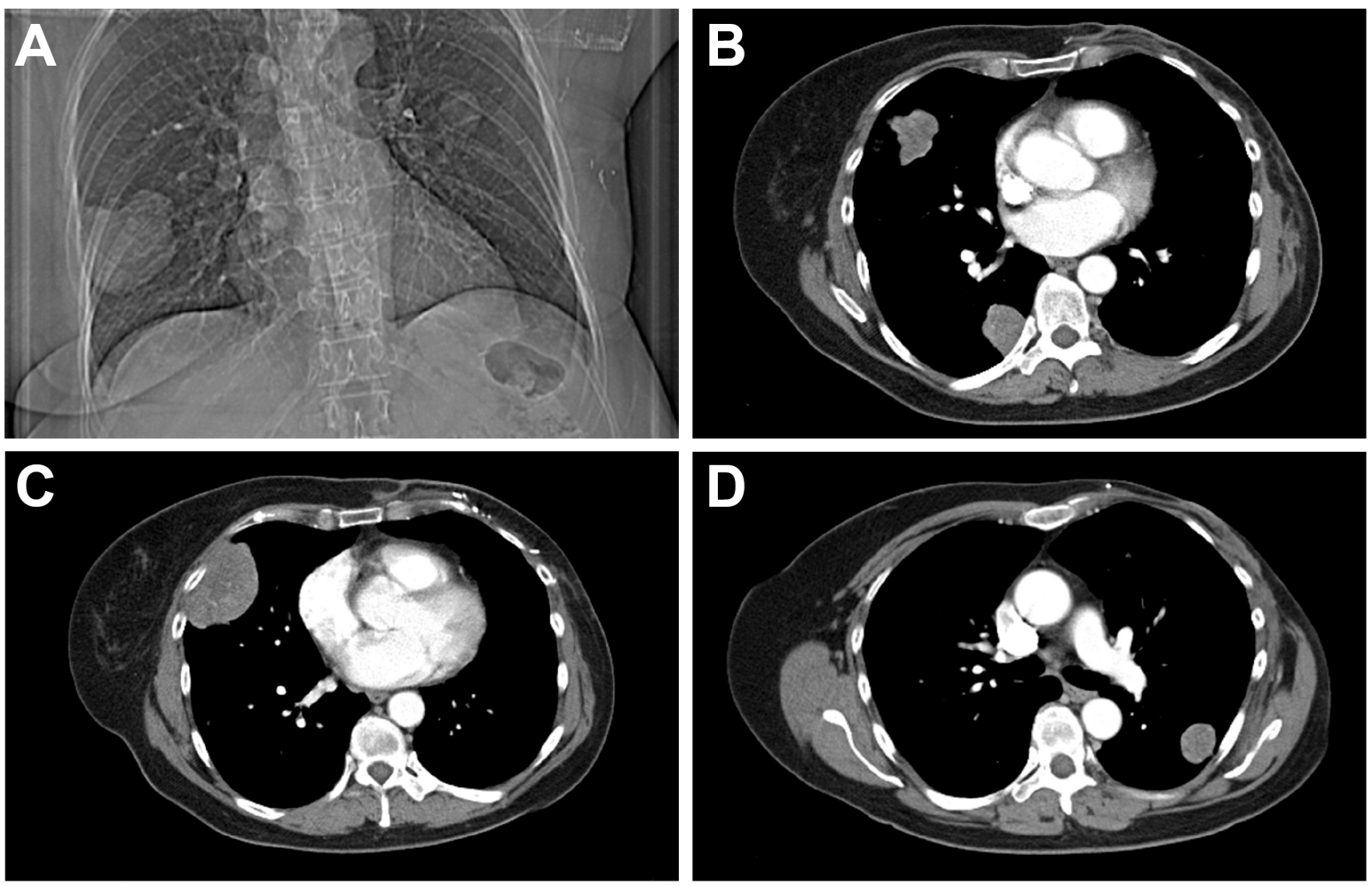

Fig. (4). (A) CT scan scout film demonstrating bilateral large pulmonary nodules consistent with metastasis. (B, C, D) Representative CT images demonstrating bilateral pulmonary metastases.

A postoperative CT scan revealed large bilateral lung metastases (Fig. 4); needle biopsy confirmed a spindle cell neoplasm consistent with metastasis. Within 5 weeks of mastectomy, she complained of headaches and a head CT scan identified an $11 \mathrm{~mm}$ right parietal lobe-enhancing lesion in the brain, consistent with a metastatic deposit. She was treated with a Doxorubicin-based chemotherapy and gamma knife radiosurgery to the brain. The disease progressed during the next six months and she expired in hospice care 8 months after her initial presentation.

\section{DISCUSSION}

Standard treatment for all PT requires a minimum of a 1 $\mathrm{cm}$ clear margin [9]. Because malignant PT rarely metastasize to regional lymph nodes [10], sentinel node biopsy is not indicated. The routine use of adjuvant chemotherapy and/or radiation therapy is not recommended for malignant PT since neither modality has shown an improvement in overall survival. In this patient, however, the size of tumor places the patient at very high risk of local recurrence, and thus adjuvant radiation therapy was considered. Moreover, she developed metastatic pulmonary and brain lesions and was offered palliative chemotherapy. The use of systemic therapy for metastatic disease of PT is based on the guidelines for treating sarcomas, not breast carcinoma. Since only the stromal component metastasizes, the metastatic deposits resemble sarcomas [11]. The incidence of metastases from malignant PT ranges from $6.2 \%[12]$ to $25 \%[3,13]$.

Malignant PT with differentiated sarcomatous components as a portion of the stromal tumor have been reported: osteosarcoma [14], liposarcoma $[15,16]$, leiomyosarcoma [17], fibrosarcoma [18], chondrosarcoma [19], rhabdosarcoma [20] and malignant fibrous histiocytoma [21]. In a review of all reported cases of metastatic PT (67 cases) prior to 1972, Kessinger and colleagues [22] noted that the average survival time after diagnosis of metastasis was 30 months. Metastatic lesions have been reported as early as at the initial diagnosis of the primary tumor and as late as 12 years after diagnosis. The longest survival time after diagnosis with metastatic disease was 14.5 years. The most common site of the initially diagnosed distant metastasis was the lungs. Other metastatic sites include the bones, liver, heart, and distant lymph nodes [23]. Rarely, direct extension without distant metastases may result in death $[24,25]$. As with soft tissue sarcomas, in general, distant pulmonary metastases may be resectable for possible cure, if it is the only site of distant disease $[4,26]$.

Malignant PT rarely metastasize to the central nervous system [27]; however, when present, widespread metastases is common. In a report by Hlavin et al., [27], the mean interval from initial diagnosis to CNS metastasis is 5 years with a range of 3.5 months and 14 years. Prognosis is universally poor with a mean survival of 29 days after diagnosis of CNS involvement system.

In summary, malignant PT represent less than $1 \%$ of all breast malignancies and commonly have an indolent clinical course with a propensity for local recurrence. Adequate histologic sampling of the stromal tissue is necessary for diagnosis of malignant PT. Rarely, malignant PT can behave very aggressively once hematogenous dissemination to the brain has been diagnosed. We present an unusual case of malignant PT with pulmonary and brain metastases treated 
with palliative mastectomy, chemotherapy, and radiosurgery to the brain.

\section{ACKNOWLEDGEMENT}

None declared.

\section{CONFLICTS OF INTEREST}

None declared.

\section{REFERENCES}

[1] Donegan WL. Sarcomas of the breast. In: Donegan WL, Spratt JS, editors. Cancer of the Breast. St. Louis, MO: Elsevier Science, Inc., 2002: 918-25

[2] Noguchi S, Yokouchi H, Aihara T, et al. Progression of fibroadenoma to phyllodes tumor demonstrated by clonal analysis. Cancer 1995; 76: 1779-85.

[3] Rowell MD, Perry RR, Hsiu JG, Barranco SC. Phyllodes tumors. Am J Surg 1993; 165: 376-9.

[4] Hart WR, Bauer RC, Oberman HA. Cystosarcoma phyllodes. A clinicopathologic study of twenty-six hypercellular periductal stromal tumors of the breast. Am J Clin Pathol 1978; 70: 211-6.

[5] Treves N, Sunderland DA. Cystosarcoma phyllodes of the breast: a malignant and a benign tumor; a clinicopathological study of seventy-seven cases. Cancer 1951; 4: 1286-332.

[6] Lester J, Stout AP. Cystosarcoma phyllodes. Cancer 1954; 7: 33553.

[7] Treves N. A study of cystosarcoma phyllodes. Ann N Y Acad Sci 1964; 114: 922-36.

[8] Macdonald OK, Lee CM, Tward JD, Chappel CD, Gaffney DK. Malignant phyllodes tumor of the female breast: association of primary therapy with cause-specific survival from the Surveillance, Epidemiology, and End Results (SEER) program. Cancer 2006; 107: 2127-33.

[9] Mangi AA, Smith BL, Gadd MA, Tanabe KK, Ott MJ, Souba WW. Surgical management of phyllodes tumors. Arch Surg 1999; 134: 487-92.

[10] Gullett NP, Rizzo M, Johnstone PA. National surgical patterns of care for primary surgery and axillary staging of phyllodes tumors. Breast J 2009; 15: 41-4.

[11] Hawkins RE, Schofield JB, Fisher C, Wiltshaw E, McKinna JA. The clinical and histologic criteria that predict metastases from cystosarcoma phyllodes. Cancer 1992; 69: 141-7.
[12] Haagensen CD. Diseases of the breast. Philadelphia: W.B. Saunders, 1975.

[13] Oberman HA. Cystosarcoma phyllodes; a clinicopathologic study of hypercellular periductal stromal neoplasms of breast. Cancer 1965; 18: 697-710.

[14] Silver SA, Tavassoli FA. Osteosarcomatous differentiation in phyllodes tumors. Am J Surg Pathol 1999; 23: 815-21.

[15] Powell CM, Rosen PP. Adipose differentiation in cystosarcoma phyllodes. A study of 14 cases. Am J Surg Pathol 1994; 18: 720-7.

[16] Qizilbash AH. Cystosarcoma phyllodes with liposarcomatous stroma. Am J Clin Pathol 1976; 65: 321-7.

[17] Isimbaldi G, Sironi M, Declich P, Galli C, Assi A. A case of malignant phyllodes tumor with muscular and fatty differentiations. Tumori 1992; 78: 351-2

[18] Suzuki-Uematsu S, Shiraishi K, Ito T, et al. Malignant phyllodes tumor composed almost exclusively of a fibrosarcomatous component: a case report and review of malignant phyllodes tumors with metastases. Breast Cancer 2010; 17: 218-24.

[19] Iihara K, Machinami R, Kubota S, Itoyama S, Sato A. Malignant cystosarcoma phyllodes tumor of the breast mainly composed of chondrosarcoma: a case report. Gen Diagn Pathol 1997; 142: 2415.

[20] Barnes L, Pietruszka M. Rhabdomyosarcoma arising within a cystosarcoma phyllodes. Case report and review of the literature. Am J Surg Pathol 1978; 2: 423-9.

[21] Hanada M, Maeda T, Takeuchi N. Cystosarcoma phyllodes of the breast with features of malignant fibrous histiocytoma. Acta Pathol Jpn 1980; 30: 91-9.

[22] Kessinger A, Foley JF, Lemon HM, Miller DM. Metastatic cystosarcoma phyllodes: a case report and review of the literature. J Surg Oncol 1972; 4: 131-47.

[23] Reinfuss M, Mitus J, Smolak K, Stelmach A. Malignant phyllodes tumours of the breast. A clinical and pathological analysis of 55 cases. Eur J Cancer 1993; 29A: 1252-6.

[24] Aronson W. Malignant cystosarcoma phyllodes with liposarcoma. Wis Med J 1966; 65: 184-7.

[25] Pietruszka M, Barnes L. Cystosarcoma phyllodes: a clinicopathologic analysis of 42 cases. Cancer 1978; 41: 1974-83.

[26] Fernandez BB, Hernanzez FJ, Spindler W. Metastatic cystosarcoma phyllodes: a light and electron microscopic study. Cancer 1976; 37 : 1737-46.

[27] Hlavin ML, Kaminski HJ, Cohen M, Abdul-Karim FW, Ganz E. Central nervous system complications of cystosarcoma phyllodes. Cancer 1993; 72: 126-30. 